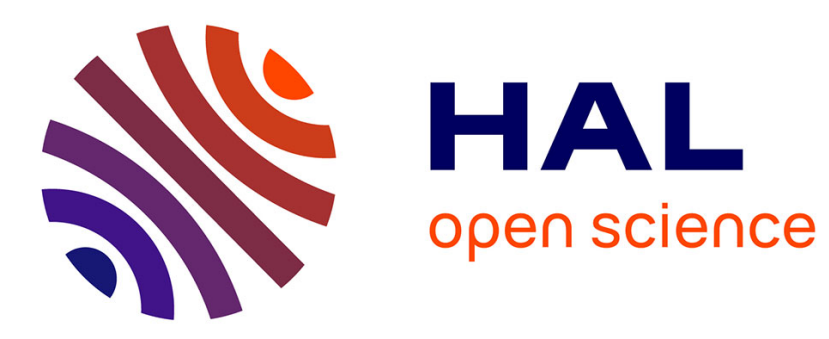

\title{
Quantum walk on a cylinder
}

Luis Bru, German J. de Valcarcel, Giuseppe Di Molfetta, Armando Pérez, Eugenio Roldán, Fernando Silva

\section{To cite this version:}

Luis Bru, German J. de Valcarcel, Giuseppe Di Molfetta, Armando Pérez, Eugenio Roldán, et al.. Quantum walk on a cylinder. Physical Review A : Atomic, molecular, and optical physics [1990-2015], 2016, 10.1103/PhysRevA.94.032328 . hal-01373413

\section{HAL Id: hal-01373413 https://hal.science/hal-01373413}

Submitted on 28 Sep 2016

HAL is a multi-disciplinary open access archive for the deposit and dissemination of scientific research documents, whether they are published or not. The documents may come from teaching and research institutions in France or abroad, or from public or private research centers.
L'archive ouverte pluridisciplinaire HAL, est destinée au dépôt et à la diffusion de documents scientifiques de niveau recherche, publiés ou non, émanant des établissements d'enseignement et de recherche français ou étrangers, des laboratoires publics ou privés. 


\title{
Quantum walk on a cylinder
}

\author{
Luis A. Bru, ${ }^{1}$ Germán J. de Valcárcel, ${ }^{2}$ Giuseppe Di Molfetta, ${ }^{3,4}$ Armando Pérez, ${ }^{3}$ Eugenio Roldán, ${ }^{2}$ and Fernando Silva ${ }^{2}$ \\ ${ }^{1}$ Optical and Quantum Communications Group, ITEAM Research Institute, Universitat Politècnica de València, Camino de Vera s/n, 46022 \\ València, Spain \\ ${ }^{2}$ Departament d'Òptica, Universitat de València, Dr. Moliner 50, 46100 Burjassot, Spain \\ ${ }^{3}$ Departamento de Fìsica Teòrica and IFIC, Universidad de Valencia-CSIC, Dr. Moliner 50, 46100 Burjassot, Spain \\ ${ }^{4}$ Aix-Marseille Université, CNRS, Laboratoire d'Informatique Fondamentale, Marseille, France
}

(Received 27 July 2016; published 28 September 2016)

\begin{abstract}
We consider the two-dimensional alternate quantum walk on a cylinder. We concentrate on the study of the motion along the open dimension, in the spirit of looking at the closed coordinate as a small or "hidden" extra dimension. If one starts from localized initial conditions on the lattice, the dynamics of the quantum walk that is obtained after tracing out the small dimension shows the contribution of several components which can be understood from the study of the dispersion relations for this problem. In fact, these components originate from the contribution of the possible values of the quasimomentum in the closed dimension. In the continuous space-time limit, the different components manifest as a set of Dirac equations, with each quasimomentum providing the value of the corresponding mass. We briefly discuss the possible link of these ideas to the simulation of high-energy physical theories that include extra dimensions. Finally, entanglement between the coin and spatial degrees of freedom is studied, showing that the entanglement entropy clearly overcomes the value reached with only one spatial dimension.
\end{abstract}

DOI: 10.1103/PhysRevA.94.032328

\section{INTRODUCTION}

Quantum walks [1-4] (QWs) refer to a variety of dynamical processes that are quantum analogs of classical random walks. As for their classical counterpart, there is a basic distinction between continuous-time and discrete-time (or coined) QWs, depending on whether time is a continuous parameter [5] or a discrete one [6], in which case a "quantum coin" is "tossed" at every step in order to decide the next state of the system. Also, both classical and QWs can evolve on a continuous space or on a lattice. An obvious (by definition) difference between classical and QWs is that quantum superpositions and interferences are inherent to the latter, which is at the root of their usefulness in quantum algorithmic and quantum information in general [7-9]. But the interest in QWs goes beyond this, as they can be understood as simulators of the Schrödinger [10,11] and Dirac equations [12-14]. Let us finally notice that some types of QWs can be implemented in relevant platforms such as cold atoms and optical networks (see $[15,16])$.

Here we study the discrete coined QW (DTQW) on a cylindric two-dimensional (2D) lattice with rectangular geometry (call it cyl-QW), namely, $\mathbb{Z} \times \mathbb{Z} / Q$, where $\mathbb{Z} / Q$ denotes the cyclic group of the integers, modulo $Q$. We note that DTQWs on simplicial complexes, including cylinders, have been introduced recently [17]. Our motivations for studying cyl-QWs are quite different. On the one hand, existing materials such as carbon nanotubes already have a cylindric geometry (with hexagonal cells in this case) so that DTQWs might capture some of the elementary physics of transport in these systems, as they actually do, to some extent, with graphene. In fact, 2D-DTQWs also exhibit an energy spectrum with conical intersections [11,18]; on the other hand, the potential fragility of a one-dimensional (1D) lattice, in which broken links forbid the walk to progress, is obviously bypassed in a cyl-QW. Moreover, the parallelism of transport on a cylinder could be more resistant to dissipation and decoherence, especially when originated from point defects. Finally, there is an especially appealing motivation for us to study the cyl-QW, which lies in its continuous limit, where space and time behave, effectively, as continuous variables. Continuous limits of QWs have been studied very many times $[10,12,19]$, showing that QWs recover the Dirac equation under proper assumptions [10-14]. From this perspective, the cyl-QW could help in modeling the effect of closed dimensions (maybe compact unobserved dimensions) on Dirac particles. As we show below in detail, in this continuous limit the existence of an unobservable closed dimension manifests as a mass term in the Dirac equation, a mass that depends on the (pseudo-)momentum of the initial state along the cyclic dimension, a situation that reminds one of the tower spectrum in Kaluza-Klein theories (see, for example $[20,21]$ ). Here, Dirac particles with different masses are selected just by the value of the momentum along the cyclic dimension (conserved by the QW evolution). Clearly this attribution of the origin of mass to the excitation of different modes in closed microscopic geometries is in very much the same spirit as in string theory and other theories that are based on the assumption of extra dimensions.

The rest of the article is organized as follows: In Sec. II A the cyl-QW is formulated. Analytical and numerical results of the dispersion relations are presented in Sec. II B. Then in Sec. III we analyze the entanglement properties of the model by deriving an analytical expression for the reduced density matrix in the long-term limit, starting from a localized initial state. In Sec. IV we compute the continuous limit of the cylQW. Our main conclusions are summarized in Sec. V.

\section{QUANTUM WALK ON A CYLINDER}

In this section we first define and characterize the alternate quantum walk (AQW) in $2 \mathrm{D}$ propagating on a cylinder. 
The corresponding dispersion relations (DR) are derived in Sec. II B. Some numerical simulations are presented to confirm the predictions made by the DR.

\section{A. Formulation}

The AQW, first introduced by Ambainis et al. in [22] for the 2D case, is the simplest way to build higher-dimensional QWs, as it makes use of a single qubit to alternate directions instead of a four-level internal state. Interestingly, it was later shown to be equivalent to the well-known Grover Walk in 2D [18,23,24] and generalized to $N$ dimensions in [18], where its dispersion relations were analyzed in detail.

We consider the quantum walker moving on a $2 \mathrm{D}$ discrete cylindrical lattice oriented along the infinite $x$ direction, with $y$ indicating the direction on the closed dimension. The total Hilbert space $\mathcal{H}$ corresponding to this system can be written as the tensor product $\mathcal{H}=\mathcal{H}_{w} \otimes \mathcal{H}_{s}$, where $\mathcal{H}_{w}$ is the Hilbert space associated to the spatial degrees of freedom with basis states $|m, l\rangle, m \in \mathbb{Z}$, and $l \in[0, Q-1]$, so that $Q$ is the number of nodes on the closed dimension. The two-dimensional Hilbert space $\mathcal{H}_{s}$ corresponds to the internal (or spin) degrees of freedom of the walker and is spanned by the states $\{|1\rangle,|-1\rangle\}$. Altogether, the basis states of $\mathcal{H}$ can be written as $|m, l ; s\rangle=|m, l\rangle \otimes|s\rangle, s= \pm 1$. The state evolution from time step $j$ to time step $j+1$ is dictated by an unitary evolution operator $\hat{U}$ so that $|\psi(j+1)\rangle=\hat{U}|\psi(j)\rangle$. For the AQW, this operator is defined as

$$
\hat{U}=\hat{S}_{y} \hat{C}_{y} \hat{S}_{x} \hat{C}_{x}
$$

being $\hat{S}_{i}$, the conditional displacement along axes $i=x, y$,

$$
\begin{gathered}
\hat{S}_{x}=\sum_{l=0}^{Q-1} \sum_{m=-\infty}^{+\infty} \sum_{s= \pm 1}|m+s, l ; s\rangle\langle m, l ; s|, \\
\hat{S}_{y}=\sum_{l=0}^{Q-1} \sum_{m=-\infty}^{+\infty} \sum_{s= \pm 1}|m, l+s(\bmod Q) ; s\rangle\langle m, l ; s|,
\end{gathered}
$$

and $\hat{C}_{i}$ the coin operator acting on the qubit, which generally can be written as

$$
\hat{C}_{i}=\left(\begin{array}{cc}
e^{i\left(\alpha_{i}+\beta_{i}\right)} \cos \theta_{i} & e^{i\left(\alpha_{i}-\beta_{i}\right)} \sin \theta_{i} \\
e^{-i\left(\alpha_{i}-\beta_{i}\right)} \sin \theta_{i} & -e^{-i\left(\alpha_{i}+\beta_{i}\right)} \cos \theta_{i}
\end{array}\right),
$$

with, in general, different angles for $i=x, y$.

The above definition for the displacement operators is equivalent to imposing periodic conditions on the wave function at site $(m, l)$ with spin component $s$, defined as $\psi_{m, l ; s}(j)=\langle m, l ; s \mid \psi(j)\rangle$. One can therefore extend the support of this function to the set $(m, l) \in \mathbb{Z}^{2}$, subject to the condition

$$
\psi_{m, l+Q ; s}(j)=\psi_{m, l ; s}(j) \forall l, j .
$$

The probability of finding the walker at point $(m, l)$ at time step $j$, regardless of the spin state, is given by

$$
P(m, l, j)=\sum_{s= \pm 1}\left|\psi_{m, l ; s}(j)\right|^{2} .
$$

We will be mostly concerned about the propagation of the walker along the open dimension $x$, thus implicitly assuming that the closed $y$ dimension is "small" as compared with the spread along the tube. In other words, we treat the propagation along $y$ as unobservable and consider only the marginal probability

$$
P(m, j)=\sum_{l=0}^{Q-1} \sum_{s= \pm 1}\left|\psi_{m, l ; s}(j)\right|^{2}
$$

\section{B. Dispersion relations}

The spectrum of any QW is an essential tool to understand its behavior [25]. Provided that the unitary operator is translationally invariant, the system can be described in terms of quasimomentum states $|k, q\rangle$, where $k$ corresponds to the $x$ direction and $q$ to the $y$ direction, respectively. Using this basis, the unitary operator (1) adopts the expression

$$
\hat{U}_{q}(k)=\left(\begin{array}{ll}
A_{11} & A_{12} \\
A_{21} & A_{22}
\end{array}\right),
$$

where

$$
\begin{aligned}
A_{11} & =e^{-i(k+q)}\left(c_{x} c_{y}+e^{2 i k} s_{x} s_{y}\right) \\
A_{12} & =e^{-i(k+q)}\left(c_{y} s_{x}-e^{2 i k} c_{x} s_{y}\right) \\
A_{21} & =e^{-i(k-q)}\left(c_{x} s_{y}-e^{2 i k} c_{y} s_{x}\right) \\
A_{22} & =e^{-i(k-q)}\left(e^{2 i k} c_{x} c_{y}+s_{x} s_{y}\right),
\end{aligned}
$$

with the notation $c_{i}=\cos \theta_{i}, s_{i}=\sin \theta_{i}$. In the latter equation, we have set all phases to zero $\alpha_{i}=\beta_{i}=0$ for $i=x, y$, since the coin angles $\theta_{x}$ and $\theta_{y}$ are the only dynamically relevant parameters. One can readily obtain the eigenvalues of $\hat{U}$, which can be written as $e^{i \omega_{ \pm}}$, where

$$
\cos \omega_{ \pm}=c_{x} c_{y} \cos (k+q)+s_{x} s_{y} \cos (k-q)
$$

defines the dispersion relations. The function $\omega_{ \pm}(k, q)$ is $2 \pi$ periodic along every component due to the discreteness of the lattice. This allows us to restrict ourselves to the first Brillouin zone $k, q \in]-\pi, \pi]$. Figure 1 shows the DR when the coin operators $\hat{C}_{i}$ are both chosen to be the Hadamard coin, i.e., $\theta_{x}=\theta_{y}=\frac{\pi}{4}$. In this twofold band structure, the most remarkable feature is the presence of conical intersections, where the two bands meet. This feature is strongly related to the propagation properties of the AQW: conical intersections are present whenever $\theta_{x}=\theta_{y}$ and they establish a mean to swap population between bands. Pure linear spreading takes place close to those points. When angles are different, $\theta_{x} \neq \theta_{y}$, the contact points disappear, thus avoiding linear spreading and allowing zero-group-velocity eigenstates, thus producing a dramatic localization of the wave function at the origin. This key feature was recently used to build an electric QW in 2D with almost perfect localization in Ref. [26]. For further details on the derivation of the DR and the influence of conical intersections we refer the reader to Refs. [18-26].

So far, we have considered the DR (9) for arbitrary values of $k$ and $q$. However, it is easy to show that cyclic conditions along the closed direction (5) restricts the possible values of $q$ to the set

$$
\left\{q_{i}=\frac{2 \pi i}{Q} \text {, where } i \in \mathbb{Z}\right\} \text {. }
$$




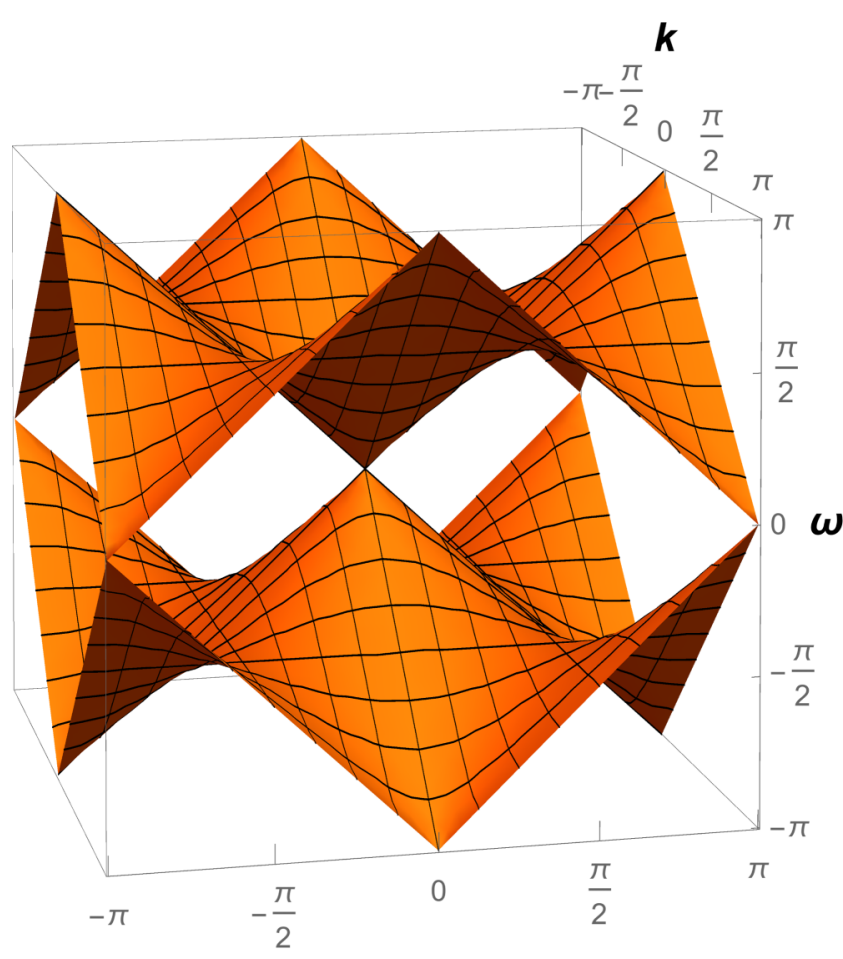

q

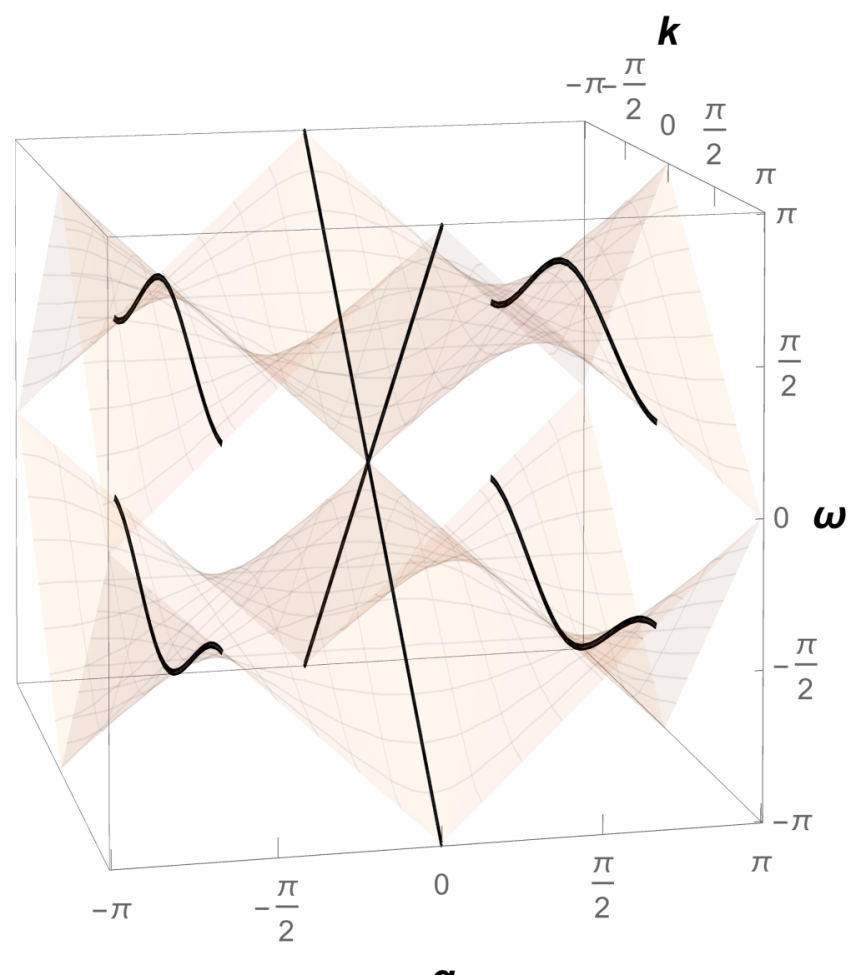

$q$

FIG. 1. Dispersion relation of the AQW in 2D for the conventional case (a) and for the cylinder with $Q=3$ nodes around cyclic dimension (b). Both of them correspond to the Hadamard case $\theta_{x}=\theta_{y}=\pi / 4$.

This set contains $Q$ different values in the first Brillouin zone, with a distribution that depends on whether $Q$ is an odd or an even number. This is due to the restrictions introduced by the

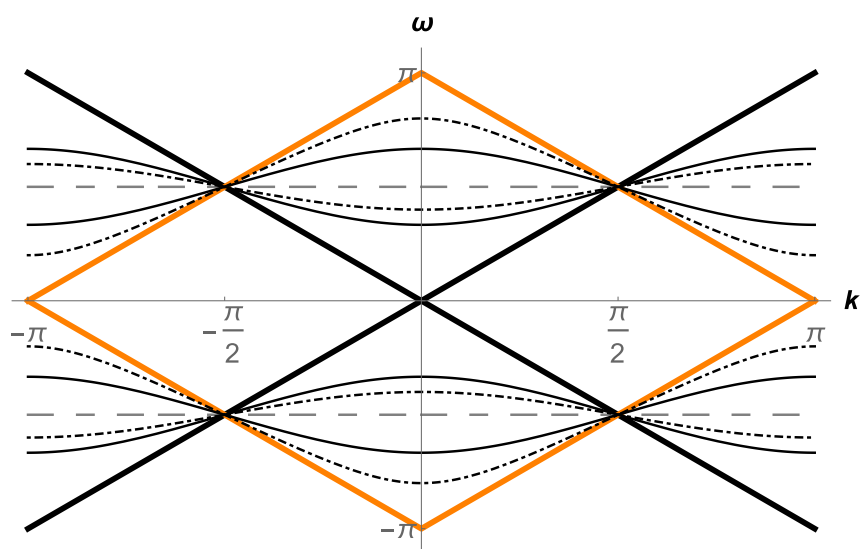

FIG. 2. Dispersion relations of the AQW on the cylinder for cases $Q=4,5$, and 6 , plotted along the quasimomentum of the open dimension $x$. The thick line (orange and black) contributions are always present, but the orange one is present only for even cases $Q=4$ and 6 . Thin solid lines correspond to $Q=6$, and the dot-dashed ones to $Q=5$. The dashed horizontal line appears only for $Q=4$.

DR symmetry properties. When $Q$ is even some degeneracies appear in the spectrum because of the symmetry of the DR; moreover, the number of degeneracies is different when $Q$ is a multiple of 4 so that the number of different discrete states appearing in the spectrum is $(Q+4) / 4$ when $Q$ is multiple of 4 and $(Q+2) / 4$ when it is not. Importantly, when $Q$ is multiple of 4 , some of the states in the spectrum become flat (see Fig. 2).

In order to interpret the derived DR, let us take a closer look to the Hadamard case. (We will restrict ourselves to this case in what follows.) Since we are mostly interested in the propagation along the open dimension $x$, the dynamics is governed by $Q$ contributions of the type

$$
\cos \omega_{ \pm}=\cos k \cos q_{i} .
$$

We notice that this formula represents a set of DRs of several 1D quantum walks [10], with each $q_{i}$ playing the role of the different $\theta$ angles of the coin operator. Therefore, we expect the $A Q W$ on the cylinder to be described by a set of several $1 D Q W$ s propagating along $x$, with different propagation velocities given by the corresponding maximum group velocities obtained from $q_{i}$. This result is one of the major results of this work and is confirmed by our numerical simulations in the following section. Moreover, we can select one or several 1D QWs by just preparing accurately the initial state in the momentum space, as in Fig. 3.

Figure 3 confirms the agreement between our numerical simulations and the prediction's bases on the DR in each case. For example, for $Q=6$ we have two different nondegenerated contributions, one of them with maximum group velocity propagation. This type of "massless" component, which is present in all cases, is stronger for even than for odd values of $Q$ due to the lower number of total contributions. The presence of this component avoids any possibility of localization of the wave function. As discussed above, this feature is ultimately due to the presence of conical intersections in the present AQW system. 


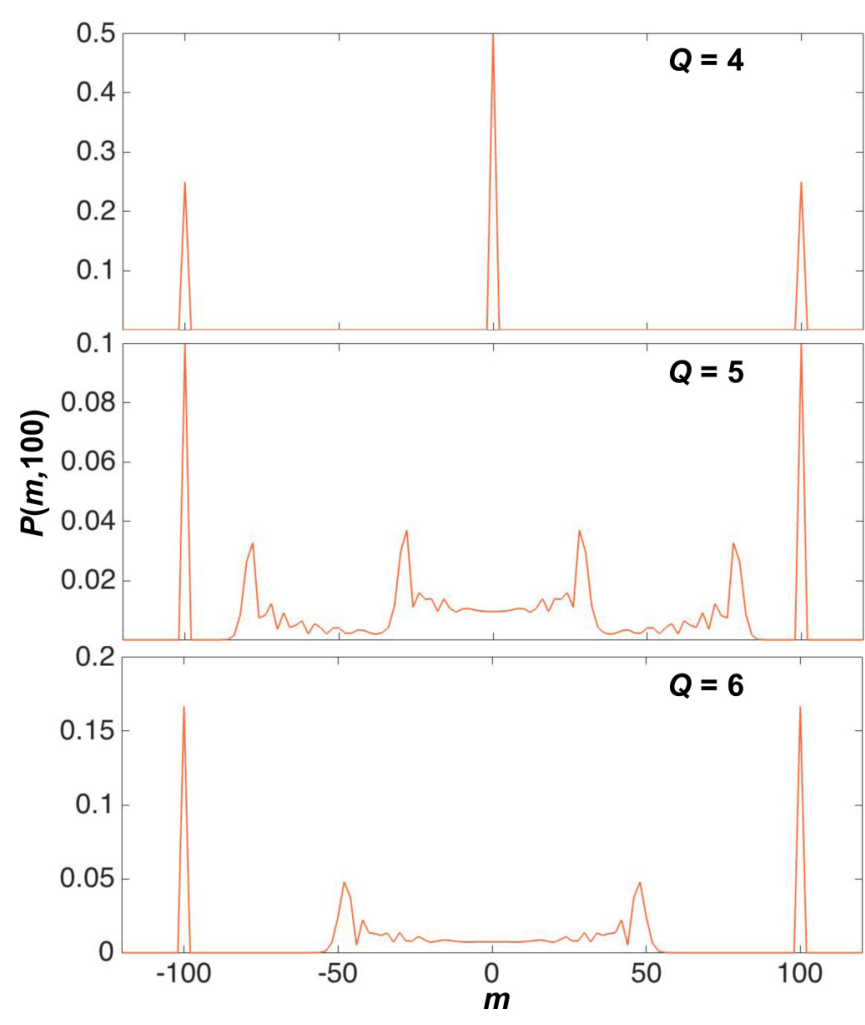

FIG. 3. Marginal probability distribution, as defined by Eq. (7) for an AQW on the cylinder in the Hadamard case after $j=100$ steps, starting from a localized initial condition $|\psi(0)\rangle=\frac{1}{\sqrt{2}}(1, i)$ at the lattice origin $(0,0)$. Different values of $Q=4,5$, and 6 are represented, from top to bottom.

On the other hand, the existence of a strictly localized component at the origin for $Q=4$ is due to the presence of a zero-group-velocity $1 \mathrm{D}$ contribution in the set. These $\omega$ flat contributions will always be present whenever $Q$ is a multiple of 4 . In all these cases, localization at the origin will show up.

\section{ENTANGLEMENT}

Entanglement between the coin and spatial degrees of freedom is generated as a consequence of the evolution of the QW [27-35]. The amount of entanglement can be quantified using the von Neumann entropy of the reduced density matrix of the coin degrees of freedom, after tracing out the spatial ones. More precisely, we define this quantity, as a function of the time step $j$, by

$$
S(j)=-\operatorname{Tr}\left\{\rho_{c}(j) \log _{2} \rho_{c}(j)\right\},
$$

where $\rho_{c}(j) \equiv \sum\langle m, l \mid \psi(j)\rangle\langle\psi(j) \mid m, l\rangle$ is the reduced density matrix for the coin space. A measure of the entanglement entropy was first numerically obtained in [27] and proven later in [30] that for a Hadamard walk with localized initial conditions the asymptotic entanglement is $S_{\text {lim }} \simeq 0.8720$ for all initial coin states, although higher values can be reached by starting from nonlocalized conditions (see also [36]). The question that arises is whether the quantum walk on a cylinder is also limited to this amount of entanglement when the evolution starts from a localized state. Our goal is to obtain an analytical expression for the reduced density matrix $\rho_{c}(j)$ in the long-term limit. This calculation is more conveniently done in the quasimomentum space and detailed in Appendix A. We consider an initial state localized at $m=0, l=0$ and arbitrary coin components, i.e.,

$$
|\psi(0)\rangle=\cos \frac{\theta}{2}|0 ; 0 ; 1\rangle+e^{i \phi} \sin \frac{\theta}{2}|0 ; 0 ;-1\rangle,
$$

where $\theta$ and $\phi$ represent the angles of the initial state on the Bloch sphere.

In Fig. 4 we have represented the asymptotic entropy of entanglement obtained from Eq. (A7) for some representative cases as a function of $\theta$ and $\phi$. In the asymptotic time behavior, the entropy derived from (A10) is maximal for $\theta=\pi / 2$ and $\phi=\frac{\pi}{2}$ or $\frac{3 \pi}{2}$, with a corresponding value:

$$
S_{\max }=\frac{2}{\pi} \log _{2} \frac{2}{\pi}+\left(1-\frac{2}{\pi}\right) \log _{2}\left(1-\frac{2}{\pi}\right) \simeq 0.945 .
$$

Such value clearly overcomes the corresponding limit with only one dimension. The differences observed in the amount of entanglement generated within the QW on a cylinder, as compared to the ordinary QW, may have important consequences. The QW has been suggested as a possible device to generate entanglement in quantum information processes [37]. On the other hand, the coin can be regarded as a thermodynamic subsystem interacting with the lattice. As such, it becomes an interesting scenario to investigate the approach to thermodynamical equilibrium in quantum systems [38]. We have shown that the QW on a cylinder behaves differently to the QW, with a dynamics that allows one to reach larger values of entanglement. Therefore, it is possible that the transition towards equilibrium will show new features. Among these features is the investigation of a non-Markovian behavior previous to the asymptotic regime, as already observed for the QW [39].

\section{THE CONTINUOUS LIMIT}

A practical tool to study the analytical properties of QWs on the discrete circle is looking at the continuous limit of the DFT of the walk [13]. In order to take the continuous limit we introduce, in the unitary operator (A3), $k=\tilde{k} \Delta x, q_{i}=\tilde{q}_{i} \Delta y$, and $t=j \Delta t$, where $(\Delta x, \Delta y, \Delta t)$ are the step sizes of the space-time lattice. Then we introduce an infinitesimal $\epsilon$ and write $\Delta t=\Delta x=\Delta y=\epsilon$, and assume that all functions are at least $C^{2}$ differentiable in all their arguments. We now expand the original discrete equations, defined by the unitary operator (A3) around $\epsilon=0$. A necessary and sufficient condition for the expression to be self-consistent at order 0 in $\epsilon$ is that $\hat{U}_{q_{i}}(k)^{(0)}=\hat{C}_{y} \hat{C}_{x}=\mathbb{I}$. This is satisfied in our AQW because we chose that angles $\alpha_{i}$ and $\beta_{i}$ are zero. If we Taylor expand each term around $\epsilon=0$, the zeroth-order terms identically vanish and the next-lowest-order contribution in $\mathrm{O}(\epsilon)$ recovers a couple of partial differential equations for the two-component wave function $\psi$. Notice that the limit is taken on both dimensions, keeping $q_{i}$ constant. A tedious but straightforward computation gives the following equation in 

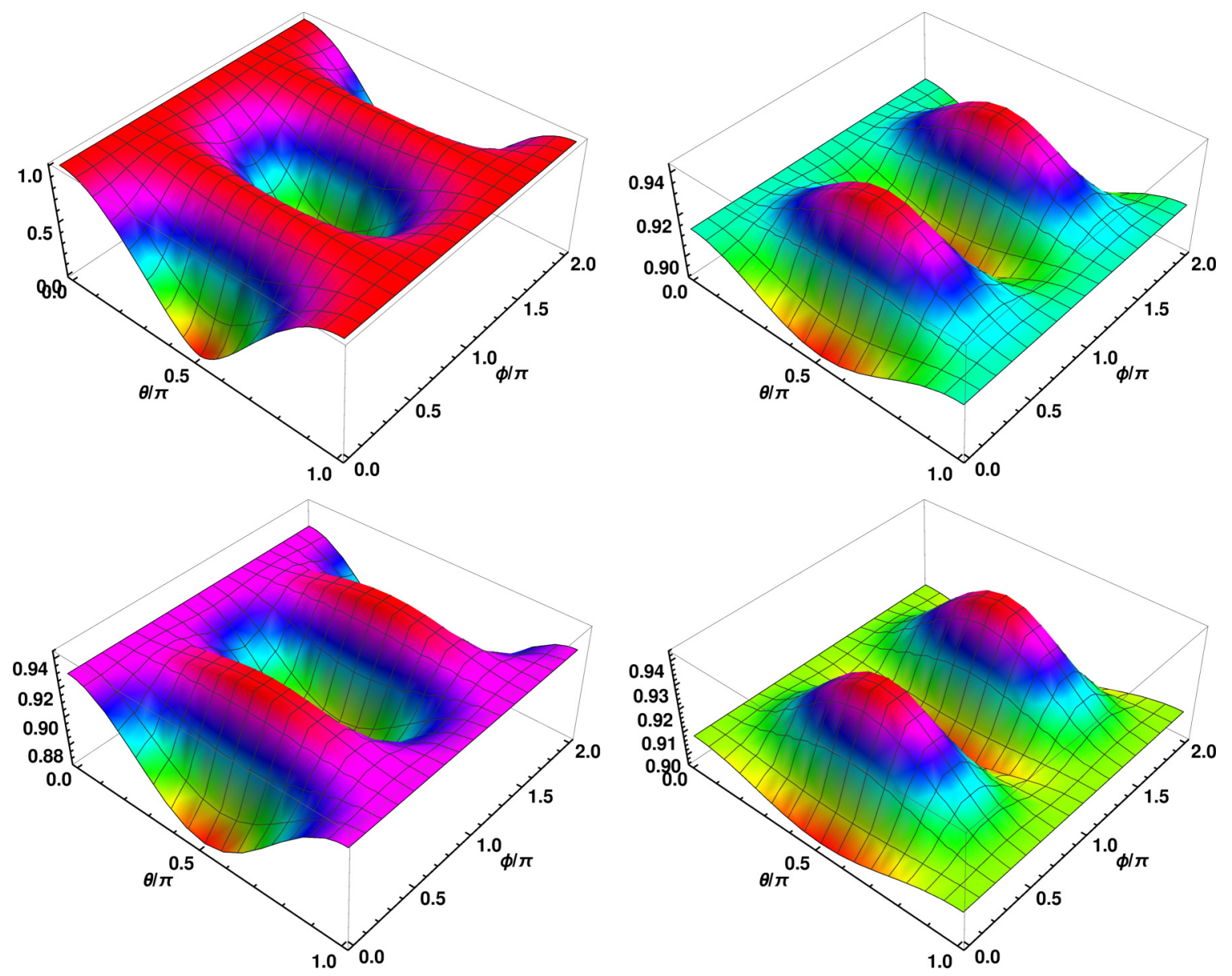

FIG. 4. Plots of the entanglement entropy for various values of the number $Q$ of nodes on the closed dimension. From left to right and top to bottom: $Q=1,5,6,7$, respectively.

physical space, obeyed by the wave function $\psi$ :

$$
\psi_{t}-\sigma_{z} \psi_{x}=i q_{i} \psi
$$

We observe that this couple of equations coincide with the massive $(1+1)$ Dirac equation, where the pseudomomentum $q_{i}$ plays the role of the fermion mass term. This is consistent with the analysis of Sec. II B. Indeed, the dispersion relation in Eq. (9) recovers the usual Dirac cone in continuous limit. In particular, this result shows that QWs on a cylinder can be used to model quantum transport of a fermion with a mass $m \in\left[0, q_{\max }\right]$, where $q_{\max }$ is the UV cutoff on the closed dimension and simulates fermions with different masses easily by a suitable choice of the initial condition.

\section{CONCLUSIONS AND OUTLOOK}

In this work we have analyzed a quantum walk defined on a cylinder. A simple approach to this problem is given by the alternated use of a single qubit on the two dimensions [23]. Although the size of the closed $y$ dimension is in principle arbitrary, it is in the spirit of this paper to regard this dimension as a sort of "hidden" or "extra" dimension, i.e., it is defined by some length scale which is much smaller than the observed spreading along the open $x$ coordinate. The reason for this approach is twofold. First, some physical devices, such as nanotubes, can be effectively described in this way. Secondly, one can establish a connection with theories in high-energy physics that assume the existence of compactified extra dimensions. In fact, the $\mathrm{QW}$ has been shown as a candidate to simulate many physical phenomena, ranging from the motion of a particle on a curved space-time [14] to Yang-Mills gauge theories [40] or neutrino oscillations $[41,42]$. In this spirit, we analyzed the motion along the open coordinate after tracing out the closed dimension. After this, the "hidden dimension" manifests in providing several components to the observed QW, which arise from different values $q_{i}$ of the quasimomentum in the closed direction. These components move each one with a different velocity, which originate from the corresponding group velocity at a given $q_{i}$.

One can get more insight about the role played by the closed dimension on the infinite one by examining the continuous limit of the QW, where a particular value $q_{i}$ is selected. One then arrives at a Dirac equation describing the motion along the open coordinate, where the pseudomomentum $q_{i}$ plays the role of the mass term. Therefore, by selecting the appropriate quasimomentum, one can easily simulate a family of Dirac equations with different masses, a situation that reminds one of the tower spectrum in Kaluza-Klein theories [20,21]. In our opinion, the simulation of the QW on a cylinder opens the possibility to investigate many aspects that appear in many high-energy theories and thus deserves further attention. 


\section{ACKNOWLEDGMENTS}

This work has been supported by the Spanish Ministerio de Educación e Innovación, MICIN-FEDER through Projects No. FPA2014-54459-P, No. FIS2014-60715-P, and No. SEV2014-0398, and by the Generalitat Valenciana through Grant No. GVPROMETEOII2014-087.

\section{APPENDIX A: ENTANGLEMENT ENTROPY}

We use the notation $\left|\psi_{k, q_{i}}(j)\right\rangle=\left\langle k, q_{i} \mid \psi(j)\right\rangle$, which represents a two-component spinor in the quasimomentum basis. With this notation we can write

$$
\left|\psi_{k, q_{i}}(0)\right\rangle=\left(\begin{array}{c}
\cos \frac{\theta}{2} \\
e^{i \phi} \sin \frac{\theta}{2}
\end{array}\right)
$$

Using the unitary operator (1), for $\theta_{x}=\theta_{y}=\frac{\pi}{4}$, in this basis, it follows that

$$
\left|\psi_{k, q_{i}}(j)\right\rangle=\hat{U}_{q_{i}}(k)\left|\psi_{k, q_{i}}(0)\right\rangle,
$$

with

$$
\hat{U}_{q_{i}}(k)=\left(\begin{array}{cc}
e^{i q_{i}} \cos k & -i e^{i q_{i}} \sin k \\
-i e^{-i q_{i}} \sin k & e^{-i q_{i}} \cos k
\end{array}\right) .
$$

The $t$ th power of $\hat{U}_{q_{i}}(k)$ is obtained from the spectral theorem:

$$
\hat{U}_{q_{i}}^{j}(k)=\sum_{h= \pm 1} e^{-i \omega_{h}\left(k, q_{i}\right) j}\left|\phi_{h}\left(k, q_{i}\right)\right\rangle\left\langle\phi_{h}\left(k, q_{i}\right)\right| .
$$

In the latter equation, $\omega_{h}\left(k, q_{i}\right)$ is obtained from the dispersion equation and $\left|\phi_{h}\left(k, q_{i}\right)\right\rangle, h= \pm 1$ are the two normalized eigenvectors of $\hat{U}_{q_{i}}(k)$, given by

$$
\left|\phi_{h}\left(k, q_{i}\right)\right\rangle=\frac{1}{N_{h}}\left(\begin{array}{c}
e^{i q_{i}} \sin k \\
-\sin q_{i} \cos k \pm \sin \omega
\end{array}\right),
$$

respectively, for $h= \pm 1$, and $N_{h}$ is an appropriate normalization constant. From the above expressions one can obtain $\rho_{c}(j)$ as

$$
\rho_{c}(j)=\frac{1}{Q} \sum_{i=0}^{Q-1} \int_{-\pi}^{\pi} \frac{d k}{2 \pi}\left|\psi_{k, q_{i}}(j)\right\rangle\left\langle\psi_{k, q_{i}}(j)\right| .
$$

Equation (A4) contains terms of the form $e^{ \pm 2 i \omega_{h} j}$. For large values of $j$, such terms become highly oscillatory, while the rest of the terms that depend on the variables $k$ and $q_{i}$ are smooth functions. We can therefore neglect the integral over $k$ of such strongly oscillatory terms. By doing so, we arrive at the expression

$$
\tilde{\rho_{c}} \equiv \lim _{j \rightarrow \infty} \rho_{c}(j)=\frac{1}{Q} \sum_{i=0}^{Q-1}\left(\begin{array}{cc}
1-r_{22}\left(q_{i}\right) & r_{12}\left(q_{i}\right) \\
r_{12}^{*}\left(q_{i}\right) & r_{22}\left(q_{i}\right)
\end{array}\right),
$$

where

$$
\begin{gathered}
r_{22}(q)=\frac{\cos ^{2} \frac{\theta}{2}[1-v(q)]+\sin ^{2} \frac{\theta}{2}[\cos 2 q+v(q)]}{2 \cos ^{2} q} \\
r_{12}(q)=\frac{e^{-2 i q} e^{i \phi}+e^{-i \phi}}{4 \cos ^{2} q}[1-v(q)] \sin \theta
\end{gathered}
$$

with $v(q) \equiv \frac{\sqrt{1-\cos (2 q)}}{\sqrt{2}}$.

As shown in Fig. 4, this magnitude can present different shapes as the value of $Q$ is changed. More importantly, we observe that one reaches values close to unity for some angles. For larger values the shape stabilizes and looks similar to the case with $Q=7$. In fact, one can derive a closed expression for $\tilde{\rho}_{c}$ in the limit $Q \rightarrow \infty$ by replacing the sum in Eq. (A7) by an integral over the continuous variable $q$, giving the final expression:

$$
\lim _{Q \rightarrow \infty} \tilde{\rho}_{c}=\frac{1}{2 \pi}\left(\begin{array}{cc}
\pi+(\pi-2) \cos \theta & {\left[e^{-i \phi}+(\pi-3) e^{i \phi}\right] \sin \theta} \\
{\left[e^{i \phi}+(\pi-3) e^{-i \phi}\right] \sin \theta} & \pi-(\pi-2) \cos \theta
\end{array}\right) .
$$

[1] J. Kempe, Contemp. Phys. 44, 307 (2003).

[2] V. Kendon, Int. J. Quantum Inform. 4, 791 (2006).

[3] N. Konno, in Quantum Potential Theory (Springer, New York, 2008), pp. 309-452.

[4] S. E. Venegas-Andraca, Quantum Inf. Process. 11, 1015 (2012).

[5] O. Mülken and A. Blumen, Phys. Rep. 502, 37 (2011).

[6] Y. Aharonov, L. Davidovich, and N. Zagury, Phys. Rev. A 48, 1687 (1993).

[7] A. Ambainis, E. Bach, A. Nayak, A. Vishwanath, and J. Watrous, in Proceedings of the Thirty-Third Annual ACM Symposium on Theory of Computing (ACM, New York, 2001), pp. 37-49.

[8] A. M. Childs, Phys. Rev. Lett. 102, 180501 (2009).

[9] N. B. Lovett, S. Cooper, M. Everitt, M. Trevers, and V. Kendon, Phys. Rev. A 81, 042330 (2010).
[10] G. J. de Valcárcel, E. Roldán, and A. Romanelli, New J. Phys. 12, 123022 (2010).

[11] M. Hinarejos, A. Pérez, E. Roldàn, A. Romanelli, and G. de Valcarcel, New J. Phys. 15, 073041 (2013).

[12] F. W. Strauch, Phys. Rev. A 73, 054302 (2006).

[13] G. Di Molfetta and F. Debbasch, J. Math. Phys. 53, 123302 (2012).

[14] G. Di Molfetta, M. Brachet, and F. Debbasch, Phys. Rev. A 88, 042301 (2013).

[15] K. Manouchehri and J. Wang, Physical Implementation of Quantum Walks (Springer Publishing Company, Inc., Cham, Switzerland, 2013).

[16] P. M. Preiss, R. Ma, M. E. Tai, A. Lukin, M. Rispoli, P. Zupancic, Y. Lahini, R. Islam, and M. Greiner, Science 347, 1229 (2015). 
[17] K. Matsue, O. Ogurisu, and E. Segawa, Quantum Inf. Process. 15, 1865 (2016).

[18] E. Roldán, C. Di Franco, F. Silva, and G. J. de Valcárcel, Phys. Rev. A 87, 022336 (2013).

[19] P. L. Knight, E. Roldán, and J. Sipe, J. Mod. Opt. 51, 1761 (2004).

[20] E. Witten, Nucl. Phys. B 186, 412(1981).

[21] V. A. Rubakov, Phys. Usp. 44, 871 (2001).

[22] A. Ambainis, J. Kempe, and A. Rivosh, in Proceedings of the Sixteenth Annual ACM-SIAM Symposium on Discrete Algorithms SODA '05 (Society for Industrial and Applied Mathematics, Philadelphia, PA, 2005), pp. 1099-1108.

[23] C. Di Franco, M. Mc Gettrick, and T. Busch, Phys. Rev. Lett. 106, 080502 (2011).

[24] C. Di Franco, M. Mc Gettrick, T. Machida, and T. Busch, Phys. Rev. A 84, 042337 (2011).

[25] A. Nayak and A. Vishwanath, arXiv:quant-ph/0010117.

[26] L. A. Bru, M. Hinarejos, F. Silva, G. J. de Valcárcel, and E. Roldán, Phys. Rev. A 93, 032333 (2016).

[27] I. Carneiro, M. Loo, X. Xu, M. Girerd, V. Kendon, and P. L. Knight, New J. Phys. 7, 156 (2005).

[28] S. Venegas-Andraca, J. Ball, K. Burnett, and S. Bose, New J. Phys. 7, 221 (2005).

[29] J. Endrejat and H. Buettner, J. Phys. A 38, 9289 (2005).
[30] G. Abal, R. Siri, A. Romanelli, and R. Donangelo, Phys. Rev. A 73, 042302 (2006).

[31] Y. Omar, N. Paunković, L. Sheridan, and S. Bose, Phys. Rev. A 74, 042304 (2006).

[32] O. Maloyer and V. Kendon, New J. Phys. 9, 87 (2007).

[33] P. K. Pathak and G. S. Agarwal, Phys. Rev. A 75, 032351 (2007).

[34] C. Liu and N. Petulante, Phys. Rev. A 79, 032312 (2009).

[35] M. Annabestani, M. R. Abolhasani, and G. Abal, J. Phys. A: Math. Theor. 43, 075301 (2010).

[36] G. J. de Valcárcel, C. Di Franco, M. Hinarejos, A. Pérez, E. Roldán, A. Romanelli, and F. Silva, in 2013 Conference on Lasers and Electro-Optics - International Quantum Electronics Conference (Optical Society of America, 2013), paper IB_P_20.

[37] S. K. Goyal and C. Chandrashekar, J. Phys. A: Math. Theor. 43, 235303 (2010).

[38] A. Romanelli, Phys. Rev. A 85, 012319 (2012).

[39] M. Hinarejos, C. Di Franco, A. Romanelli, and A. Pérez, Phys. Rev. A 89, 052330 (2014).

[40] P. Arnault, G. D. Molfetta, M. Brachet, and F. Debbasch, Phys. Rev. A 94, 012335 (2016).

[41] A. Mallick, S. Mandal, and C. M. Chandrashekar, arXiv:1604.04233.

[42] G. Di Molfetta and A. Pérez, arXiv:1607.00529. 